\title{
WATER ENTRY OF A WEDGE INTO WAVES IN THREE DEGREES OFFREEDOM
}

\author{
Shi Yan Sun \\ Jiangsu University of Science and Technology, China \\ Hai Long Chen \\ Harbin Engineering University, China \\ Gang Xu \\ Jiangsu University of Science and Technology, China
}

\begin{abstract}
The hydrodynamic problem of a two-dimensional wedge entering into a nonlinear wave in three degrees of freedom is investigated based on the incompressible velocity potential theory. The problem is solved through the boundary element method in the time domain. To avoid numerical difficulties due to an extremely small contact area at the initial stage, a stretched coordinate system is used based on the ratio of the Cartesian system in the physical space to the distance travelled by the wedge in the vertical direction. The mutual dependence of body motion and wave loading is decoupled by using the auxiliary function method. Detailed results about body accelerations, velocities and displacements at different Froude numbers or different waves are provided, and the mutual effect between body motion and wave loading is analysed in depth.
\end{abstract}

Keywords: Water entry; nonlinear waves; three degrees of freedom; boundary element method; auxiliary function method.

\section{INTRODUCTION}

The impact between wave and structure occurs very often in violent sea conditions. When a ship advances in rough seas, the bow may emerge from the water and then re-enter into the wave with high speed. In those cases, the local area of hull surface would surfer a very large impact pressure, and a great risk would be imposed to the structure. The wave/ structure impact can also be observed in problems of wave energy converters operating in rough seas, large waves hitting an offshore platform, and liquid sloshing in a tank. Those phenomena are dynamically equivalent to a wedge entering freely into a wave.

The past research often focused on the wedge entry into calm water. Wagner's theory was commonly used to analyse this problem, especially for bodies with small deadrise angles.
Most popular works on the subject include those by: Howison et al. [1] for bodies with small deadrise angles, Faltinsen [2] on the wedge with finite deadrise angle, and Korobkin et al. [3] for the body with elastic deformation. In contrast to Wagner's theory, the fully nonlinear boundary element method is more accurate in simulating such a nonlinear process. Dobrovolskaya [4] and Zhao \& Faltinsen [5] considered the vertical entry of a wedge into water. Semenov \& Iafarati [6] solved the problem of vertical entry of an asymmetric wedge, while $\mathrm{Xu}$ et al. [7] investigated the case of oblique entry of an asymmetric wedge. The problem of twin wedges was analysed by $\mathrm{Wu}$ [8]. Wu et al. [9] considered the vertical entry of a wedge in free fall motion with a single degree of freedom, and Xu et al. [10] considered the free fall with three degrees of freedom, in which the vertical motion is coupled with the horizontal and rotational motion. 
Wave slamming is a common phenomenon but rarely considered in the research. Faltinsen [11] was the first to investigate the slamming effect of a wedge advancing in waves. In his work, the slamming pressure of ship section is calculated based on Wagner's theory. Sun et al. [12] adopted the boundary element method to investigate the problem of a wedge entering into waves in a given motion. In that work, the mutual dependence between wave and body motion is not considered, however, this coupling effect plays a significant role in modelling the physical process of wedge entering into waves.

In this paper, the coupled process of a wedge entering into waves in free motion is investigated. The boundary element method is adopted, and the fully nonlinear boundary conditions are imposed on the free surface and the instantaneous body surface. Some analyses associated with the Froude number and wave effect are undertaken. On the one hand, unlike the calm water cases, the effect of Froude number is changed noticeably by the waves. The reason for this is that the fluid flow velocity distribution is altered in the wave field, and thus free surface profiles, pressure distributions and motions under different Froude numbers reveal some peculiar features. On the other hand, the effects of wave height and wave number which are closely related to wave effects are studied in depth. It should be noted that the reported investigations mainly focus on slamming effects and motion traits during the short interval when the slamming occurs.

\section{MATHEMATICAL MODEL AND NUMERICAL PROCEDURE}

\section{PHYSICAL PARAMETERS AND CARTESIAN COORDINATE SYSTEM}

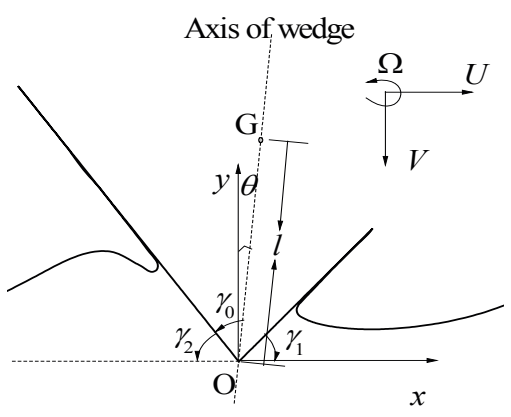

Fig. 1. Sketch of the problem

The 2-D problem considered here is illustrated in Fig. 1, which shows the wedge entering into the wave in three degrees of freedom. The Cartesian coordinate system $O-x y$ with the origin $O$ fixed at the wedge tip is defined so that the $x$-axis points horizontally and the $y$-axis points upwards. The horizontal, vertical and rotational velocities about the gravity centre $G$ are denoted as $U, V$ and $\Omega$, respectively.
$V$ is positive when pointing downwards, while $\Omega$ when anti-clockwise. $\theta$ is the heel angle measured from the wedge symmetry axis to the $y$ axis. As the wedge rotates, $\theta$ varies with time. $\gamma_{0}$ is the angle between the symmetry axis and the surface of the wedge. The water density $\rho$, the initial vertical velocity $V_{0}$, and the distance $l$ between the wedge's tip and centre of gravity are used for non-dimensionalization. In this case, the Froude number is defined as $F_{r}=V_{0} / \sqrt{g l}$, in which $g$ is the acceleration of gravity.

The deadrise angles $\gamma_{1}$ and $\gamma_{2}$ on the right and left hand sides, respectively, can be obtained from the following relationship

$$
\gamma_{1}=\frac{\pi}{2}+\theta-\gamma_{0}, \gamma_{2}=\frac{\pi}{2}-\theta-\gamma_{0}
$$

During the water entry, the vertical distance $s$, the horizontal distance $h$, and the heel angle $\theta$ of the travelling wedge can be obtained through

$s(t)=\int_{0}^{t} V(\tau) d \tau, \quad h(t)=\int_{0}^{t} U(\tau) d \tau \quad \theta(t)=\theta_{0}+\int_{0}^{t} \Omega(\tau) d \tau$

where $\theta_{0}$ is the initial heel angle at the moment of water entry. The displacement of wedge tip in the vertical direction can be written as

$$
s_{P}=-s(t)+\cos \theta-\cos \theta_{0}
$$

and in the horizontal direction it becomes

$$
h_{P}=h(t)+\sin \theta-\sin \theta_{0}
$$

\section{GOVERNING EQUATION AND BOUNDARY CONDITIONS}

When the fluid is assumed incompressible and inviscid and the flow is irrotational, the velocity potential $\phi$ can be introduced. This potential satisfies the Laplace equation in the fluid domain

$$
\nabla^{2} \phi=0
$$

and its gradient is equal to the fluid velocity. In the body-fixed system, the dynamic and kinematic boundary conditions on the instantaneous free surface $S_{\mathrm{F}}$ can be written in Lagrangian form as

$$
\frac{D \phi}{D t}=\frac{1}{2} \nabla \phi \cdot \nabla \phi-\frac{1}{F_{r}}\left(y-s+\cos \theta-\cos \theta_{0}+A_{0}\right)
$$


$\frac{D x}{D t}=\frac{\partial \phi}{\partial x}-U-\Omega \cos \theta, \frac{D y}{D t}=\frac{\partial \phi}{\partial y}+V+\Omega \sin \theta$

where $A_{0}$ is the wave elevation at the moment of water entry. On the wetted surface $S_{0}$ of the body the impermeable boundary condition takes the form

$$
\frac{\partial \phi}{\partial n}=(U-\Omega Y) n_{x}+(-V+\Omega X) n_{y}
$$

where $\mathbf{n}=\left(n_{x}, n_{y}\right)$ is the normal vector to the body surface pointing out of the fluid domain, and $\mathbf{X}=(X, Y)$ is the position vector relative to the centre of gravity or the rotating centre $G$. The negative sign before $V$ is due to the fact that $V$ is positive when it is downwards. At a sufficiently large distance from the body, the disturbance is assumed to have diminished, and there is only the incident potential. The velocity of fluid tends to that due to the undisturbed potential $\phi_{1}$, and the far field boundary condition can therefore be written as

$$
\nabla \phi \rightarrow \nabla \phi_{\mathrm{I}}
$$

At $t=0$, the initial condition can be expressed as

$y(x, t=0)=\eta_{\mathrm{I}}(x, t=0), \quad \phi(x, \eta, t=0)=\phi_{\mathrm{I}}\left(x, \eta_{\mathrm{I}}, t=0\right)$

At the initial stage of water entry, there is only a tiny part of the body immersed in the wave, and in order to maintain sufficient numerical accuracy, extremely small elements are needed. If a fixed fluid domain is used in the physical coordinate system $O-x y$, the choice of the truncated boundary should take into account the disturbed area at later stages. All this would lead to a large number of elements at the initial stage. To solve this problem, a stretched system $O-\alpha \beta$ proposed by Wu et al. [9] can be used here. The distance $s$ travelled by the wedge in the vertical direction is set as the stretched ratio between the physical system and the stretched system. At the initial stage we can write

$\phi(x, y, t)=s \varphi_{\mathrm{D}}(\alpha, \beta, t)+\phi_{\mathrm{I}}(x, y, t), \alpha=x / s, \beta=y / s$

in which $\varphi_{\mathrm{D}}$ is the velocity potential due to the disturbance in the stretched system. The body surface boundary condition can then be written as

$$
\frac{\partial \varphi_{\mathrm{D}}}{\partial n}=(U-\Omega Y) n_{\alpha}+(-V+\Omega X) n_{\beta}-\frac{\partial \phi_{\mathrm{I}}}{\partial n}
$$

where $\nabla \varphi_{\mathrm{D}} \rightarrow 0$ at a sufficiently large distance from the body. It should be pointed out that the normal derivatives of $\varphi_{\mathrm{D}}$ and $\phi_{\mathrm{I}}$ in Eq. (12) are taken from the stretched and
Cartesian coordinate systems respectively. At $t=0, \varphi_{\mathrm{D}}=0$ on the free surface, or $\phi=\phi_{\mathrm{I}}$. Substituting Eq. (11) into Eqs. (6) and (7), we have

$$
\begin{aligned}
& \frac{D\left(s \varphi_{\mathrm{D}}+\phi_{\mathrm{I}}\right)}{D t}=\frac{1}{2}\left[\left(\varphi_{\mathrm{D} \alpha}+\phi_{\mathrm{Ix}}\right)^{2}+\left(\varphi_{\mathrm{D} \beta}+\phi_{\mathrm{Iy}}\right)^{2}\right]-\frac{1}{F_{r}}\left(s \beta-s+\cos \theta-\cos \theta_{0}+A_{0}\right) \\
& \frac{D(s \beta)}{D t}=\phi_{\mathrm{D} \beta}+\varphi_{\mathrm{I} y}+V+\Omega \sin \theta, \frac{D(s \alpha)}{D t}=\phi_{\mathrm{D} \alpha}+\varphi_{\mathrm{Ix}}-U-\Omega \cos \theta
\end{aligned}
$$

The disturbance potential $\varphi_{\mathrm{D}}$ can be updated together with the free surface. In the subsequent time steps we can define $\phi(x, y, t)=s \varphi(\alpha, \beta, t)$. The body surface boundary condition for $\varphi$ retains the same form as that in Eq. (8)

$$
\frac{\partial \varphi}{\partial n}=(U-\omega Y) n_{\alpha}+(-V+\omega X) n_{\beta}
$$

The free surface boundary conditions can be written as

$$
\frac{D(s \varphi)}{D t}=\frac{1}{2}\left(\varphi_{\alpha}^{2}+\varphi_{\beta}^{2}\right)-\frac{1}{F_{r}}\left(s \beta-s+\cos \theta-\cos \theta_{0}+A_{0}\right)
$$

$$
\frac{D(s \beta)}{D t}=\varphi_{\beta}+V+\Omega \sin \theta, \frac{D(s \alpha)}{D t}=\varphi_{\alpha}-U-\Omega \cos \theta
$$

It should be noted that the reason for using Eq. (11) at $t=0$ is to avoid $\varphi$ which tends to infinity as $S \varphi$ equals the incident potential on the free surface and at a large distance from the body at $t=0$.

\section{THE BOUNDARY ELEMENT METHOD}

Through Green's identity, the Laplace equation in the fluid domain can be converted into the integral equation over the whole boundary

$$
A(p) \phi(p)=\int\left(\ln r_{\mathrm{pq}} \frac{\partial \phi(q)}{\partial n_{\mathrm{q}}}-\phi(q) \frac{\partial \ln r_{\mathrm{pq}}}{\partial n_{\mathrm{q}}}\right) d l_{\mathrm{q}}
$$

where $A(p)$ is the solid angle of boundaries at point $p$, and $r_{p q}$ is the distance from the field point $p$ to the source point $q$. Straight line elements with variables assumed to vary linearly within each element are distributed along the boundary. To perform the integration over each element in Eq. (18), we define

$$
\mathbf{r}=\alpha \mathbf{i}+\beta \mathbf{j}=\sum_{i=1}^{2} h^{i}(u) \cdot \mathbf{r}^{i}, \quad \phi=\sum_{i=1}^{2} h^{i}(u) \cdot \phi^{i}
$$

in which $\mathbf{r}$ is the position vector from the origin, with $\mathbf{i}$ and $\mathbf{j}$ being the unit vectors in the $\alpha$ and $\beta$ directions, respectively. The shape functions are chosen as 


$$
h^{1}(u)=1-u, \quad h^{2}(u)=u
$$

where $0 \leq u \leq 1$. Substituting Eqs. (20) and (21) into (19), we have

$A(p) \phi(p)=\sum_{j=1}^{N_{e}} \int_{0}^{1} \ln r_{\mathrm{pq}} \sum_{i=1}^{2} h^{i}(u) \frac{\partial \phi_{j}^{i}(q)}{\partial n} l d u-\sum_{j=1}^{N_{e}} \int_{0}^{1} \frac{\partial \ln r_{\mathrm{pq}}}{\partial \mathrm{n}} \sum_{i=1}^{2} h^{i}(u) \phi_{j}^{i}(q) l d u$

where $N_{e}$ is the total number of elements. The integrations within each element can be obtained through the analytical solution given by $\mathrm{Lu}, \mathrm{He} \& \mathrm{Wu}[13]$.

\section{THE PRESSURE}

Based on the Bernoulli equation, the pressure can be written as

$p=-\left[\phi_{t}+\frac{1}{2} \nabla \phi \cdot \nabla \phi+\frac{1}{F_{r}}\left(y-s+\cos \theta-\cos \theta_{0}+A_{0}\right)\right]$

At each time step, the velocity potential $\phi$ can be solved through the numerical scheme given in the previous section, from which its gradient $\nabla \phi$ can be obtained. However, the temporal derivative of potential $\phi_{t}$ is still unknown explicitly. To deal with this problem, the auxiliary function method is adopted $[14,15]$. Notice that the potential $\phi_{t}$ also satisfies the Laplace function in the fluid domain. The normal derivative of $\phi_{t}$ on the body surface can be written as [16]

$$
\frac{\partial \phi_{t}}{\partial n}=(\dot{\mathbf{U}}+\dot{\boldsymbol{\Omega}} \times \mathbf{X}) \cdot \mathbf{n}-\mathbf{U} \cdot \frac{\partial \nabla \phi}{\partial n}+\boldsymbol{\Omega} \cdot \frac{\partial}{\partial n}[\boldsymbol{X} \times(\mathbf{U}-\nabla \phi)]
$$

where $\mathbf{U}=U \mathbf{i}+V \mathbf{j}, \boldsymbol{\Omega}=\boldsymbol{\Omega} \mathbf{k}$ with $\mathbf{k}=\mathbf{i} \times \mathbf{j}$, and the dot means the temporal derivative. Particular attention should be paid to accelerations in Eq. (22), which are unknown before the force is found. To decouple their nonlinear mutual dependence, we define

$\phi_{t}=\chi_{0}+s \dot{U} \chi_{1}+s \dot{V} \chi_{2}+s \dot{\Omega} \chi_{3}-\mathbf{U} \cdot \nabla \phi+\boldsymbol{\Omega} \cdot \boldsymbol{X} \times(\mathbf{U}-\nabla \phi)$

Here $\chi_{i}(i=0, \cdots 3)$ satisfy the Laplace equation. The body surface boundary conditions for the auxiliary functions can be written as

$$
\begin{aligned}
& \frac{\partial \chi_{0}}{\partial n}=0 \\
& \frac{\partial}{\partial} \\
& \frac{\partial \chi_{2}}{\partial n}=n_{\beta}
\end{aligned}
$$

$$
\frac{\partial \chi_{3}}{\partial n}=\left(X n_{\beta}-Y n_{\alpha}\right)
$$

Based on the Bernoulli equation, and considering zero pressure on the free surface, we have

$$
\begin{gathered}
\chi_{0}=-\left[\frac{1}{2}|\nabla \varphi|^{2}+\frac{1}{F r^{2}}\left(s \beta-s+\cos \theta-\cos \theta_{0}+A_{0}\right)\right] \\
+U \varphi_{\alpha}-V \varphi_{\beta}-\Omega \cdot\left[X \cdot\left(-V-\varphi_{\beta}\right)-Y \cdot\left(U-\varphi_{\alpha}\right)\right] \\
\chi_{i}(i=1,2,3)=0
\end{gathered}
$$

In the far field, the disturbance potential is assumed to have sufficiently diminished, and there is only the incident potential. Consequently, the boundary conditions can be written as

$$
\begin{gathered}
\frac{\partial \chi_{0}}{\partial n}=\frac{\partial \phi_{\mathrm{It}}}{\partial \mathrm{n}}+U \frac{\partial \phi_{\mathrm{Lx}}}{\partial n}-V \frac{\partial \phi_{\mathrm{IV}}}{\partial n}-\Omega \cdot \frac{\partial}{\partial n}\left[X \cdot\left(-V-\phi_{y}\right)-Y \cdot\left(U-\phi_{x}\right)\right] \\
=\frac{\partial \phi_{\mathrm{It}}}{\partial \mathrm{n}}+(U-Y \Omega) \frac{\partial \phi_{\mathrm{Lx}}}{\partial n}-(V-X \Omega) \frac{\partial \phi_{\mathrm{Iy}}}{\partial n}-\Omega \cdot\left[n_{x} \cdot\left(-V-\phi_{\mathrm{Iy}}\right)-n_{y} \cdot\left(U-\phi_{\mathrm{Ix}}\right)\right] \\
\frac{\partial \chi_{i}}{\partial n}(i=1,2,3)=0
\end{gathered}
$$

The above auxiliary functions can also be solved through the boundary element method discussed in Sec. 2.3. Once they are all found, $\phi_{t}$ can be obtained and used in Eq. (22) for pressure calculation.

\section{COUPLED MOTION}

The Newton's equation of motion for a body in waves can be written as

$$
[\mathbf{M}][\mathbf{A}]=[\mathbf{F}]+\left[\mathbf{F}_{e}\right]
$$

where

$[\mathbf{M}]=\left[\begin{array}{ccc}m & 0 & 0 \\ 0 & m & 0 \\ 0 & 0 & I\end{array}\right],[\mathbf{A}]=\left[\begin{array}{c}\dot{U} \\ -\dot{V} \\ \dot{\Omega}\end{array}\right],[\mathbf{F}]=\left[\begin{array}{l}F_{1} \\ F_{2} \\ F_{3}\end{array}\right],\left[\mathbf{F}_{e}\right]=\left[\begin{array}{c}0 \\ -m g \\ 0\end{array}\right]$

In Eq. (34), $m$ is the mass of the two dimensional wedge of unit length, and $I$ denotes the rotational inertia about the centre of gravity. The matrix $[\mathbf{F}]$ is the hydrodynamic force, which can be obtained through integrating the pressure from Eq. (22) along the wetted surface of the body. Thus we have

$$
F_{i}=s \cdot \int_{S_{0}} p n_{i} d S
$$


where $\left(n_{1}, n_{2}, n_{3}\right)=\left(n_{\alpha}, n_{\beta}, X n_{\beta}-Y n_{\alpha}\right)$ Based on the definition of the auxiliary functions in Eq. (24), the hydrodynamic force can be divided into two parts: one part containing the terms associated with unknown accelerations, and the other part containing the rest of the hydrodynamic force which has been solved through the numerical scheme given in the previous section. In this case, the unknown accelerations can be obtained through solving the uncoupled equation of motion with unknown terms moved to the left hand side of Eq. (33). Thus we have

$$
[\mathbf{M}+\mathbf{C}][\mathbf{A}]=[\mathbf{Q}]+\left[\mathbf{F}_{e}\right]
$$

where $[\mathbf{C}]$ is the matrix with the coefficients

$$
C_{i j}=s^{2} \cdot \int_{S_{0}} \chi_{i} n_{j} d S
$$

and the coefficients of matrix $[\mathbf{Q}]$ can be obtained as

$$
\begin{aligned}
Q_{i}= & -s \cdot \int_{S_{0}}\left\{\chi-\left(U \varphi_{\alpha}-V \varphi_{\beta}\right)+\Omega \cdot\left[X \cdot\left(-V-\varphi_{\beta}\right)-Y \cdot\left(U-\varphi_{\alpha}\right)\right]\right. \\
& \left.+\frac{1}{2} \nabla \varphi \cdot \nabla \varphi+\frac{1}{F r^{2}}\left(s \beta-s+\cos \theta-\cos \theta_{0}+A_{0}\right)\right\} \cdot n_{i} d S
\end{aligned}
$$

\section{NUMERICAL RESULTS AND DISCUSSION}

The wave environment in the above procedure can be changed according to the specific problem. In the reported case, the $5^{\text {th }}$ order Stokes wave for deep water is adopted. Based on the manner described before for Eq. (1), the nondimensional potential and wave elevation can be written as [17]

$$
\begin{aligned}
\phi_{1}= & \frac{1}{F_{r}} \sqrt{\frac{1}{k^{3}}}\left(k A e^{k y_{0}} \sin \theta-\frac{1}{2} k^{3} A^{3} e^{k y_{0}} \sin \theta+\frac{1}{2} k^{4} A^{4} e^{2 k y_{0}} \sin 2 \theta\right. \\
& \left.-\frac{37}{24} k^{5} A^{5} e^{k y_{0}} \sin \theta+\frac{1}{12} k^{5} A^{5} e^{3 k y_{0}} \sin 3 \theta\right) \\
\eta_{\mathrm{I}}= & A\left[\left(1-\frac{3}{8} k^{2} A^{2}-\frac{422}{384} k^{4} A^{4}\right) \cos \vartheta+\left(\frac{1}{2} k A+\frac{1}{3} k^{3} A^{3}\right) \cos 2 \vartheta\right. \\
& \left.+\left(\frac{3}{8} k^{2} A^{2}+\frac{297}{384} k^{4} A^{4}\right) \cos 3 \vartheta+\frac{1}{3} k^{3} A^{3} \cos 4 \vartheta+\frac{125}{384} k^{4} A^{4} \cos 5 \vartheta\right]
\end{aligned}
$$

where

$$
\begin{gathered}
\vartheta=k x_{0}-\omega t+\vartheta_{0} \\
\omega=\frac{\sqrt{k}}{F_{r}}\left(1+\frac{1}{2} k^{2} A^{2}+\frac{1}{8} k^{4} A^{4}\right)
\end{gathered}
$$

and $\vartheta_{0}, \omega$, and $k$ are, respectively, the initial phase, wave circular frequency, and wave number. It should be noted that $A$ is not the wave amplitude but $H / 2$, in which $H$ is the wave height.

\section{COMPARISON}

The case of a symmetric wedge entering into calm water vertically through free fall motion is used for comparison. Fig. 2 shows the time-histories of accelerations obtained numerically from the present procedure and by Wu (2004) and $\mathrm{Xu}$ et al. (2010), as well as the experimental results recorded by $\mathrm{Wu}$ (2004). The rotational inertia $I$ is set equal to $45 \mathrm{kgm}^{2}$, the distance between the wedge tip and the centre of gravity is $l=0.25$, and the included angle is $\gamma_{0}=\pi / 4$. The deadrise angles $\gamma_{1}$ and $\gamma_{2}$ of the symmetric wedge at $t=0$ are both $\pi / 4$, and the initial heel angle is $\theta_{0}=0$. This angle will not change for the case with free fall motion. The acceleration of gravity with frictional resistance deducted is denoted as $g_{e}$. It can be seen from Fig. 2 that the vertical accelerations obtained from the present procedure are very close to those obtained by Wu (2004) and Xu et al. (2010), and this implies that the present numerical methodology is accurate. In the next cases, unless specified otherwise, $\gamma_{0}$ and $\theta_{0}$ are set equal to $\pi / 4$ and 0 , respectively.
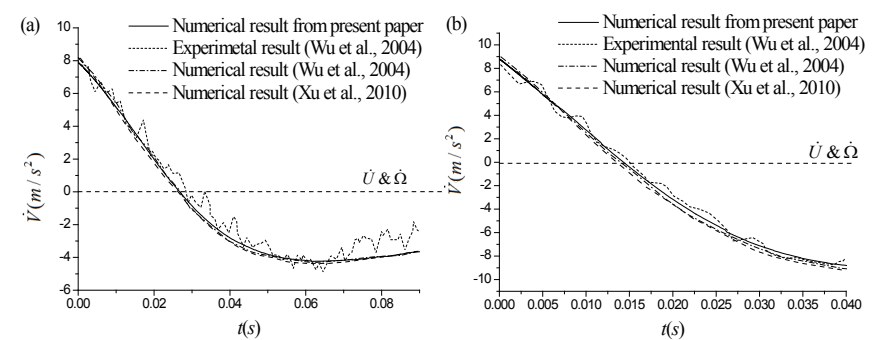

Fig. 2. Time-histories of acceleration for symmetric wedge entering water through free fall motion: (a) ge $=8.0062 \mathrm{~ms}^{-2}, M_{b}=13.522 \mathrm{kgm}^{-1}$ $V_{0}=0.95623 \mathrm{~ms}^{-1}$. (b) $\mathrm{ge}=8.9716 \mathrm{~ms}^{-2}, M_{b}=30.188 \mathrm{kgm}^{-1}, V_{0}=1.69673 \mathrm{~ms}^{-1}$.

\section{WATER ENTRY OF THE WEDGE INTO THE WAVE AT DIFFERENT FROUDE NUMBERS}

Based on the dynamic boundary condition given by Eq. (6), it can be found that when the Froude number is larger, the gravity effect is less prominent. The same conclusion can also be made from the pressure equation (22). A limiting case is when the Froude number tends to infinity, and in this case the gravity effect is negligible. If the Froude number is larger, the disturbance potential is also larger, based on Eq. (11). It can also be found that the importance of the wave potential in the total potential becomes weaker, as proved by Eq. (39). To study the effect of the Froude number, the wedge with the mass $1 \mathrm{~kg} / \mathrm{m}$ and rotational inertia $45 \mathrm{kgm}^{2}$ is released to hit the wave crest freely at different initial velocities: $3 \mathrm{~m} / \mathrm{s}, 6 \mathrm{~m} / \mathrm{s}$ and $9 \mathrm{~m} / \mathrm{s}$. The wave height and the wave length are respectively set equal to $0.8 \mathrm{~m}$ and $6 \mathrm{~m}$. Based on the nondimensionalized procedure applied to Eq. (1), the corresponding Froude numbers are 1.92, 3.83, and 5.75. At $t=0$, we have $\theta_{0}=0$ and $\gamma_{0}=\pi / 4$. Fig. 3 gives the free surface profiles and pressure distributions when the wedge has travelled the vertical distance $s=0.4$. Based on the discussion by Sun et al. [12], when the water is not calm 
and the wedge enters into the wave vertically, the results are not symmetric above the wedge axis due to the effect of the horizontal wave velocity component. However, based on the former discussion, it has been found that increasing the Froude number would make the wave effect weaker. Thus in Fig. 3, the free surface profiles and pressure distributions on the two hand sides of the wedge become more and more symmetric as the Froude number increases. A limiting case is when the Froude number tends to infinity. In this case the wave effect is negligible and the results on the two hand sides become completely symmetric.

Figs. 4-6 give the time-histories of acceleration, velocity and displacement for the wedge entering into the wave in three degrees of freedom with different Froude numbers. As shown in Fig. 4(a), when the Froude number is smaller, the absolute value of vertical deceleration $\dot{V}$ in non-dimensional sense is also smaller. This coincides with the results in Fig. 5(a), in which the absolute value of vertical velocity decreases more slowly with the decreasing Froude number. Thus in Fig. 6(a), the vertical distance travelled by the wedge with a smaller Froude number is larger due to smaller deceleration and larger velocity in vertical direction. This phenomenon seems to be in contradiction with the general idea that a body moves faster when the initial velocity or Froude number is larger. In fact, during the short interval of impact action, the body with a larger Froude number also suffers a larger impact pressure, which prevents the wedge from going down quickly. This can also be explained through the existence of a wave. The wedge with a smaller Froude number suffers a more unbalanced pressure distribution, see Fig. 3(b). Consequently, the hydrodynamic force in the vertical direction becomes smaller, and this directly leads to a smaller deceleration in vertical direction and larger accelerations in other directions. Thus, the accelerations in Fig. 4(b) and (c) with smaller Froude numbers are noticeably larger. Correspondingly, the velocities and displacements in Figs. 5 and 6 (b) and (c) increase as the Froude number decreases.
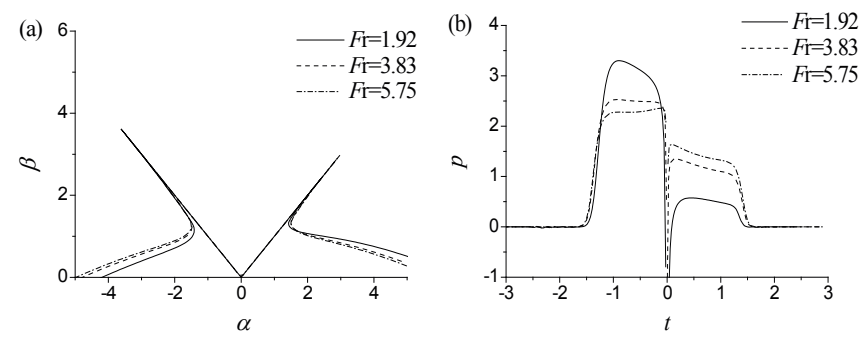

Fig. 3. Free surface profiles (a) and pressure distributions (b) for wedge entering into wave at different Froude numbers $(s=0.4)$.
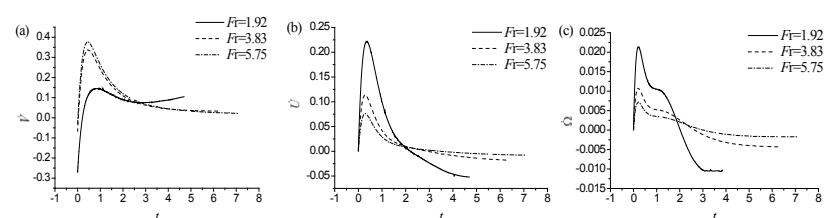

Fig. 4. Time-histories of (a) vertical, (b) horizontal, and (c) rotational acceleration for wedge entering into wave at different Froude numbers
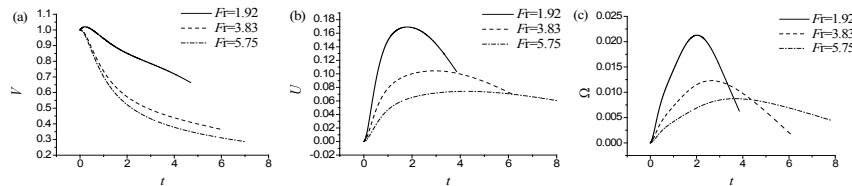

Fig. 5. Time-histories of (a) vertical, (b) horizontal, and (c) rotational velocity for wedge entering into wave at different Froude numbers
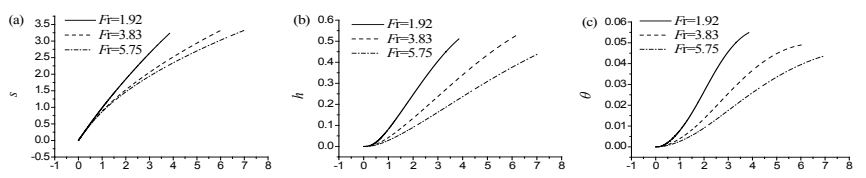

Fig. 6. Time-histories of (a) vertical, (b) horizontal, and (c) rotational displacement for wedge entering into wave at different Froude numbers

\subsection{Water entry of the wedge into different waves}

As can be seen from Eq. (39), the flow motion due to incident wave is proportional to $A \sqrt{k} / F_{\mathrm{r}}$. It is evident that when $A \sqrt{k} / F_{\mathrm{r}}<<1$, the wave effect can be neglected. If $A \sqrt{k} / F_{\mathrm{r}}$ is not a small number and the Froude number $F_{\mathrm{r}}$ is fixed, the wave effect depends on the wave height $H=2 \mathrm{~A}$ and the wave number $k$. Two limiting cases are $H=0$ and $k=0$, both corresponding to calm water conditions.

Fig. 7 gives the free surface profiles and pressure distributions for the wedge hitting the wave crests with different heights, at a fixed vertical travelled distance $s=0.4$. As $H$ increases, the wave slope becomes increasingly steeper and the pressure distributions on the two hand sides become more and more unbalanced. The reason for this is that the horizonal velocity of the wave increases with $H$ and the difference between the fluid velocity distributions on the two hand sides becomes more and more prominent. Fig. 8 gives the accelerations for the wedge hitting the wave crests with different heights. It is evident that as $H$ increases, the absolute value of vertical acceleration decreases, while the horizontal and rotational accelerations increase noticeably. This can be explained through the pressure distribution in Fig. 7(b). The wave effect becomes increasingly prominent as $H$ increases. As a result, the pressure on the seaward side becomes larger while that on the other side becomes smaller. This directly leads to the increase of the hydrodynamic force in the vertical direction and its decrease in other directions. As $H$ decreases, the pressures on the two hand sides become gradually more symmetric, the hydrodynamic force in the vertical direction increases while decreasing in other directions. The limiting case is $H \rightarrow 0$, in which the wave effect is negligible and the hydrodynamic forces and accelerations in the horizontal and rotational directions also decrease to zero. 

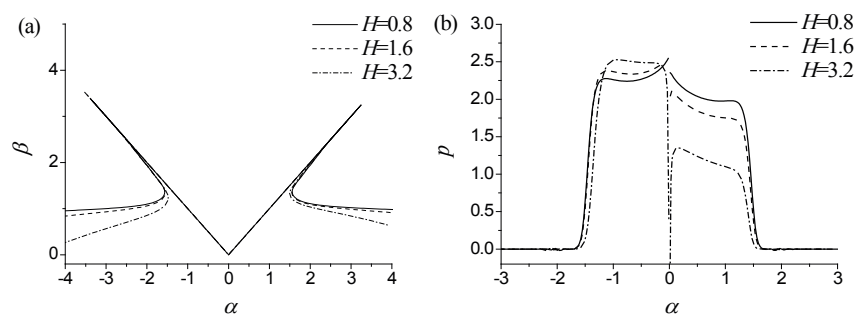

Fig. 7. Free surface profiles (a) and pressure distributions $(b)$ for wedge entering into waves with different wave heights $(s=0.4)$
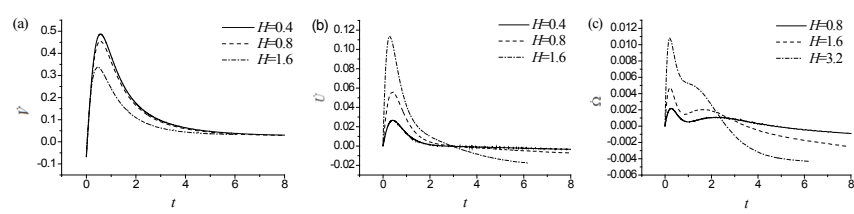

Fig. 8. Time-histories of (a) vertical, (b) horizontal, and (c) rotational acceleration for wedge entering into waves with different wave heights

Fig. 9 gives the free surface profiles and pressure distributions for the wedge hitting the wave crests with different wave numbers $k$, at the vertical distance travelled by the wedge $s=0.4$. As $k$ decreases, the wave effect becomes less prominent and therefore the pressure distributions on the two hand sides become more and more symmetric. In fact, estimating the wave effect only through $k$ and $h$ is not precise, as the effective deadrise angle between the wave and the wedge surface, as well as the wave velocity distributions in different phases are also very important for impact pressure [12]. In such a case, the pressure distribution in Fig. 9 shows some peculiar features, for example, the largest pressure is at $k=0.17$, and this is because smaller $k$ provides a smaller deadrise angle. Fig. 10 gives the accelerations for the wedge hitting the wave crests with different numbers $k$. It is evident that as $k$ increases, the absolute value of vertical acceleration decreases. This is attributed to the smaller pressure in Fig. 9(b), and the deeper reason for this is that a larger $k$ corresponds to larger deadrise angle on the two hand sides, see Fig. 9(a). As the wave number $k$ increases, the horizontal acceleration in Fig. 10(b) decreases while the rotational acceleration in Fig. 10(c) increases. It should be noted that after a short time interval of slamming, the motion of the wedge is fully driven by the wave and its accelerations mainly depend on the velocity distribution and the slope of the wave in the wave field.

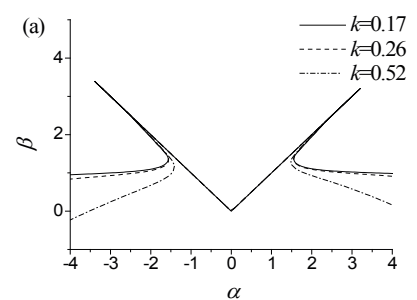

$\alpha$

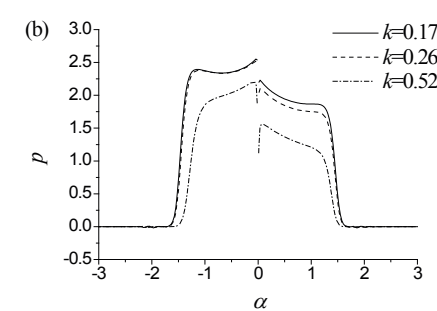

Fig. 9. Free surface profiles (a) and pressure distributions (b) for wedge entering into waves with different wave numbers $(s=0.4)$

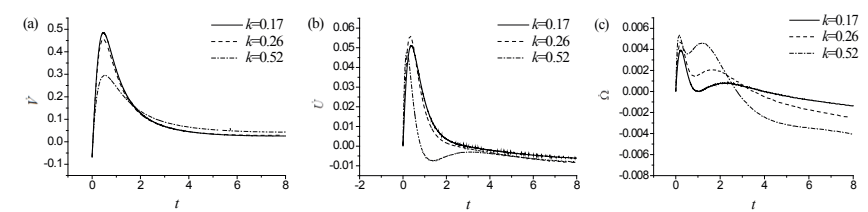

Fig. 10. Time-histories of (a) vertical, (b) horizontal, and (c) rotational acceleration for wedge entering into waves with different wave numbers

\section{CONCLUSIONS}

The problem of water entry of a wedge into waves in three degrees of freedom has been investigated using the fully nonlinear boundary element method based on the velocity potential theory. Extensive simulations have been undertaken for a wedge entering into the Stokes wave with a given initial vertical velocity, from which the following conclusions can be drawn:

(1) Except the calm water conditions, in all other cases the modes of wedge motion in the wave field vary greatly depending on the Froude number. As the Froude number increases, the absolute value of vertical acceleration increases, while the horizontal and rotational accelerations decrease. The reason for this is that the velocity distribution in the fluid field is noticeably changed by the wave. In this unsteady flow field, the Froude number is larger and the wave effect is less prominent. Thus we can conclude that it is not rational to neglect the wave effect absolutely in real ocean engineering problems, unless the Froude number is sufficiently large. In other words, calm water slamming is only a limiting case of the wave entry problem.

(2) When a wedge hits the peak of the Stokes wave with changing height at a given initial Froude number, a larger wave height will give a larger horizontal velocity in the fluid field, which will increase the pressure on the seaward side of the wedge and decrease on the back side. The wedge will surfer a more unbalanced pressure distribution, and this will lead to smaller acceleration in the vertical direction and larger in other directions.

(3) The effect of wave number is more complex. This is mainly due to the fact that the fluid field is affected by many other factors when the wave number $k$ changes, for instance, the relative location and effective deadrise angle between the wedge and the wave.

(4) The present work analyses the impact between a wedge and water wave in a broad context, including the coupled effect. It has many practical implications, such as ship advancing in rough seas, wave interaction with coastal structures, and hostile wave hitting an offshore platform.

\section{ACKNOWLEDGEMENTS}

This work is supported by the National Natural Science Foundation of China (Grants No. 51579052, 51309125, 
51579120) and the National Natural Science Foundation of Jiangsu (BK20151327).

\section{REFERENCES}

1. S.D. Howison, J.R. Ockendon, S.K, Wilson, Incompressible water-entry problems at small deadrise angles, J. Fluid Mech., 222, 215-230, 1991.

2. O.M. Faltinsen, Water entry of a wedge with Finite deadrise angle, J. Ship Res., 46(13), 39-51, 2002.

3. A. Korobkin, R. Guéret, Š. Malenica, Hydroelastic coupling of beam finite element model with Wagner theory of water impact, J. Fluids Struct., 22(4), 493-504, 2006.

4. Z.N. Dobrovolskaya, On some problems of similarity flow of fluid with a free surface, J. Fluid Mech., 36(4), 805-829, 1969.

5. R. Zhao, O. Faltinsen, Water entry of two-dimensional bodies, J. Fluid Mech., 246, 593-612, 1993.

6. Y.A. Semenov, A. Iafrati, On the nonlinear water entry problem of asymmetric wedges, J. Fluid Mech., 547, 231256, 2006.

7. G.D. Xu, W.Y. Duan, G.X. Wu, Numerical simulation of oblique water entry of asymmetrical wedge, Ocean Eng., 35(16), 1597-1603, 2008.

8. G.X. Wu, Numerical simulation of water entry of twin wedges, J. Fluids Struct., 22, 99-108, 2006.

9. G.X. Wu, H. Sun, Y.S. He, Numerical simulation and experimental study of water entry of a wedge in free fall motion, J. Fluids Struct., 19(3), 277-289, 2004.

10. G.D. Xu, W.Y. Duan, G.X. Wu, Simulation of water entry of a wedge through free fall in three degrees of freedom, Math. Phys. \& Eng. Science, 466, 2219-2239, 2010.

11. O.M. Faltinsen, Sea loads on ships and offshore structures, Cambridge University Press, 1990.

12. S.Y. Sun, S.L. Sun, G.X Wu, Oblique water entry of a wedge into waves with gravity effect, J. Fluids Struct., 52(1), 49-64, 2015.

13. C.H. Lu, Y.S. He, G.X. Wu, Coupled analysis of nonlinear interaction between fluid and structure during impact, J. Fluids Struct., 14(1), 127-146, 2000.

14. G.X. Wu, R. Eatock Taylor, Transient motion of a floating body in steep waves, $11^{\text {th }}$ Workshop on Water Waves and Floating Bodies, Hamburg, Germany, 1996.
15. G.X. Wu, R. Eatock Taylor, The coupled finite element and boundary element analysis of nonlinear interactions between waves and bodies, Ocean Eng., 30(3), 387-400, 2003.

16. G.X. Wu, Hydrodynamic force on a rigid body during impact with liquid, J. Fluids Struct., 12(5), 549-559, 1998.

17. J.D. Fenton, A fifth-order Stokes theory for steady waves, J. Waterway Port Coastal and Ocean Eng., 111, 216-234, 1985.

\section{CONTACT WITH THE AUTHORS}

\author{
Shi Yan Sun \\ e-mail:shiyan_sun@126.com
}

Jiangsu University of Science and Technology Mengxi Road, 212003 Zhenjiang

China

Hai Long Chen

e-mail:chenhailong@hrbeu.edu.cn

Harbin Engineering University

Nantong Street, 150001 Harbin

CHINA

\section{Gang Xu}

e-mail:g.xu@just.edu.cn

Jiangsu University of Science and Technology Mengxi Road, 212003 Zhenjiang

China 